\title{
The Cost-Effectiveness of Expanded Testing for Primary HIV Infection
}

\author{
Andrew Coco, MD, MS \\ Healthcare Research Center, Lancaster \\ General Hospital, Lancaster, Penn
}

Conflict of interest: none reported

\section{CORRESPONDING AUTHOR}

Andrew Coco, MD, MS

Director, Healthcare Research Center

Comprehensive Care Clinic for HIV Disease

Family and Community Medicine

Lancaster General Hospital

555 N Duke St

Lancaster, PA 17604-3555

ascoco@lancastergeneral.org

\begin{abstract}
PURPOSE Primary infection with the human immunodeficiency virus (HIV) is a major factor in the HIV epidemic. Most patients become symptomatic and seek care, but seldom are they tested or is their condition diagnosed. The objectives of this study are to determine whether it is cost-effective to expand testing for primary HIV infection to a larger cohort of patients, and, if so, which diagnostic assay is most cost-effective.
\end{abstract}

METHODS We undertook a cost-effectiveness analysis of testing a hypothetical cohort of more than 3 million outpatients with fever and other viral symptoms regardless of HIV risk factors using 3 diagnostic assays: p24 antigen enzyme immunosorbent assay (EIA), HIV-1 RNA assay, and third-generation HIV-1 EIA. Antiretroviral therapy was started when the CD4 cell count decreased to 350/ $\mu \mathrm{L}$. Outcome measures were the incremental cost-effectiveness of the diagnostic assays, number of cases identified, cases avoided in sexual partners, and threshold prevalence. For sensitivity analyses, we used $\$ 50,000$ as the threshold for cost-effectiveness.

RESULTS At the baseline prevalence of $0.66 \%$, p24 antigen EIA testing was the most cost-effective option at a cost of $\$ 30,800$ per quality-adjusted life-year gained when compared with no testing. There were 17,054 cases identified, and infection was avoided in 435 partners. Probabilistic sensitivity analysis, in which the estimates for all variables are varied simultaneously, determined that expanded testing with p24 antigen EIA compared with no testing had a $67 \%$ probability of being costeffective at the baseline prevalence and a $71 \%$ probability at a prevalence of $1 \%$.

CONCLUSIONS Expanded testing for primary HIV infection with p24 antigen EIA may be a sound expenditure of health care resources.

Ann Fam Med 2005;3:391-399. DOI: 10.1370/afm. 375.

\section{INTRODUCTION}

$\mathrm{P}$ rimary human immunodeficiency virus (HIV) infection is a transient, symptomatic illness characterized by high HIV-1 RNA levels before an effective immune response develops. ${ }^{1}$ More than $90 \%$ of cases go undiagnosed even though up to $90 \%$ of patients have symptoms and seek medical care. ${ }^{2,3}$ Primary HIV infection plays a major role in the HIV epidemic. ${ }^{4}$

Diagnosing primary HIV infection allows for an important opportunity to interrupt HIV transmission, because persons in this stage of HIV infection can be sources of new infections. ${ }^{4,5}$ First, they are in a state of heightened infectivity because they have high levels of viremia, often with a molecule count exceeding 1,000,000/ $\mathrm{LL} .{ }^{1}$ Additionally, because affected persons are unaware of the diagnosis, they might not practice preventive sexual behavior. Many patients who learn of being HIV-infected adopt behaviors that can reduce the risk for transmitting HIV. ${ }^{6}$ In addition to the public health benefits of early diagnosis, patients can also benefit by being observed for immune function deterioration and given timely initiation of antiretroviral therapy based on clinical practice guideline recommendations. ${ }^{7}$

There are numerous reasons why $90 \%$ of cases of primary HIV infec- 
tion are undiagnosed. Primarily, the symptom complex is similar to that of influenza and other nonspecific viral illnesses. ${ }^{1}$ Several studies have concluded that no symptom is sufficiently sensitive or specific to allow for targeted testing. ${ }^{8,9}$ Second, it is difficult to determine who has a high probability of primary HIV infection when evaluating risk factors alone. ${ }^{10}$ Some patients may be unaware of their risky behavior. ${ }^{11}$ Health care workers can further contribute to the problem by failing to ask about risk factors, or they might feel unqualified to manage issues associated with HIV infection. ${ }^{3}$

Confusion about which test to order can also contribute to the low detection rate of primary HIV infection. Sensitivity, specificity, and cost limit the available options. At the initial stages of infection, HIV-1 antibody tests are nonreactive, and the diagnosis of primary HIV infection is made by either the p24 core antigen test or HIV-1 RNA assays (viral load). The p24 core antigen test is more specific and less expensive than the HIV-1 RNA assays, but it is less sensitive in detecting cases. The HIV-1 RNA assays are highly sensitive, but they are more expensive and have decreased specificity, which can result in a higher false-positive rate when used in groups that have a low disease prevalence. Another option is the third-generation HIV-1 antibody test, which is able to detect immunoglobulin (Ig) M as well as IgG antibodies. The Panel on Clinical Practices for Treatment of HIV Infection through the US Department of Health and Human Services (DHHS) recommends testing with the HIV-1 RNA assay when acute infection is suspected and risk factors are present but advises against testing lower risk populations because of concerns about false-positive diagnoses. ${ }^{12}$ Given the low percentage of primary HIV infection diagnoses, however, it is unlikely that many clinicians follow these guidelines and do any testing. ${ }^{2}$

Because of the differences in characteristics and costs of the available diagnostic assays, a cost-effectiveness analysis of expanded testing of persons with viral symptoms at varying prevalence rates could be useful for policy development and guiding clinical practice. The objectives of this study were to perform an incremental cost-effectiveness analysis of expanded testing of persons with viral symptoms and at least 1 risk factor using these 3 diagnostic assays for primary HIV infection and to determine the lowest prevalence at which expanded testing is cost-effective.

\section{METHODS}

\section{Study Design}

The study was an incremental cost-effectiveness analysis of 3 tests for primary HIV infection using a decision analytical model. A strategy of no testing was used for baseline comparison, because little testing for this diag- nosis is currently done. The analysis included the following outcome measures of expanded testing for primary HIV infection: (1) increased quality-adjusted life-years (QALYs) in the patient as a result of starting antiretroviral therapy before profound deterioration in immune function occurs; and (2) prevention of HIV infection in the patient's sexual partners. Several potential outcome measures were not included: (1) benefits of immunizations (hepatitis A and B and Streptococcus pneumoniae), cervical cancer screening, and tuberculosis screening (2) $^{2}$ prevention of HIV infection in needle-sharing contacts of injection drug users; (3) avoidance of costly diagnostic workups and hospitalization for those with more severe primary HIV infection symptoms; and (4) benefits of detecting cases of chronic HIV infection. Starting antiretroviral treatment during the acute phase of HIV was not included because data showing a benefit for structured treatment interruptions in preserving natural immunity were lacking. ${ }^{13}$ The harms from expanded testing included in the analysis were (1) decreased quality of life caused by the anxiety resulting from a false-positive diagnosis, and (2) decreased quality of life associated with being aware of being HIV infected.

The time frame for the study was 39.9 years, the average life expectancy of the mean age (39.5 years) of the hypothetical cohort being tested. ${ }^{14,15}$ The analysis adopted a societal perspective, including all costs and health effects, except for work loss and transportation, which were considered negligible compared with laboratory testing, visit costs, and lifetime medical costs for treatment of HIV disease. The analysis also provided information from the perspective of a third party payer by determining the cost per case of primary HIV infection diagnosed. We conducted sensitivity analyses to determine the stability of the results using reasonable variations in the data and assumptions.

\section{Target Population}

A national estimate of the prevalence of primary HIV infection in symptomatic ambulatory patients regardless of risk factors is published in this issue of Annals. ${ }^{16}$ The estimated primary HIV infection prevalence for patients with a fever was $0.66 \%$ (95\% confidence interval $[\mathrm{CI}], 0.53 \%-0.92 \%)$, for those with a rash it was $0.56 \%(95 \% \mathrm{CI}, 0.35 \%-0.94 \%)$, and for those with a sore throat it was $0.13 \%(95 \% \mathrm{CI}, 0.10 \%-0.19 \%)$. The highest of these rates $(0.66 \%)$ was used as the baseline for the analysis. To make population projections about the number of cases diagnosed, number of infections avoided, and program costs, we assumed that 20,000, or $50 \%$ of the $40,000^{18,19}$ newly infected patients annually, developed symptoms and sought care. At the baseline prevalence of $0.66 \%, 3,030,303$ patients would need to be tested in the model. 
The prevalence of primary HIV infection has also been directly measured in a study of patients at an urban urgent care center who complained of viral symptoms and had at least 1 HIV risk factor. ${ }^{17}$ The prevalence in this study was $1 \%$ (95\% CI, 0.1\%-1.9\%). This prevalence was used to explore how a wider range of values affected the analysis.

\section{Diagnostic Tests}

Three tests with considerably different characteristics are available: (1) HIV-1 RNA assay (by polymerase chain reaction, branched-chain DNA, or transcription-mediated amplification, which is $100 \%$ sensitive but has a false-positive rate of $2 \%$ and is more expensive; (2) p24 antigen enzyme immunosorbent assay (EIA), which is less sensitive but has a false-positive rate of almost $0 \%$ and a moderate cost; and (3) third-generation HIV-1 EIA, which is less sensitive and specific than the other 2 tests, but costs the least. This test, also known as an HIV-1 antigen sandwich or combitest, ${ }^{8,9}$ is capable of detecting both IgG and IgM antibodies. Standard HIV-1 antibody EIA tests detect only IgG antibody and are much less sensitive for primary HIV infection. A rapid test (OraQuick HIV rapid test) became available in late 2002 that could identify HIV-infected persons earlier and decrease the failure rate for return visits for test results. This test was not included in the analysis because we lacked data in the setting of primary HIV infection testing.

\section{Study Model}

The study model is a decision analytical model (Figure 1) in which a hypothetical cohort of 3,030,303 million patients with fever and other viral symptoms consistent with primary HIV infection, regardless of risk factors, were tested by either the p24 antigen EIA, an HIV-1 RNA assay, a third-generation HIV-1 EIA, or not tested at all. In the model sufficient serum was collected initially so that a standard HIV-1 antibody test and, if needed, a confirmatory Western blot reflexively test could be done for those with positive initial tests. All patients were scheduled for a return visit in 14 days for test results and posttest counseling; a projected followup rate was $69 \% .^{20,21}$ Patients with a positive test had follow-up HIV clinical care arranged, including transportation, if necessary. After testing, patients were categorized through Bayesian revision according to test result: true positive, false positive, true negative, or false negative.

\section{Event Pathways}

Patients in the true-positive category who continued care were seen for an initial visit at 1 month, at which time the standard HIV antibody test and Western blot assay would be positive. ${ }^{22}$ At this visit, an initial battery of laboratory tests (Table 1), as well as a CD4 cell count and a quantitative HIV-1 RNA assay, was ordered. ${ }^{28}$ The cost of genotype resistance testing was not included in the baseline laboratory work, but it was added, as reflected in the higher range of values used in the sensitivity analysis (Table 1). Continued care entailed a return visit, a CD4 cell count determination, and a quantitative HIV-1RNA assay every 4 months. ${ }^{7}$ Surveillance visits continued for 6.2 years, the mean duration of time it takes from diagnosis for the CD4 cell count reach $350 / \mu \mathrm{L} .{ }^{29}$ At this stage of infection,

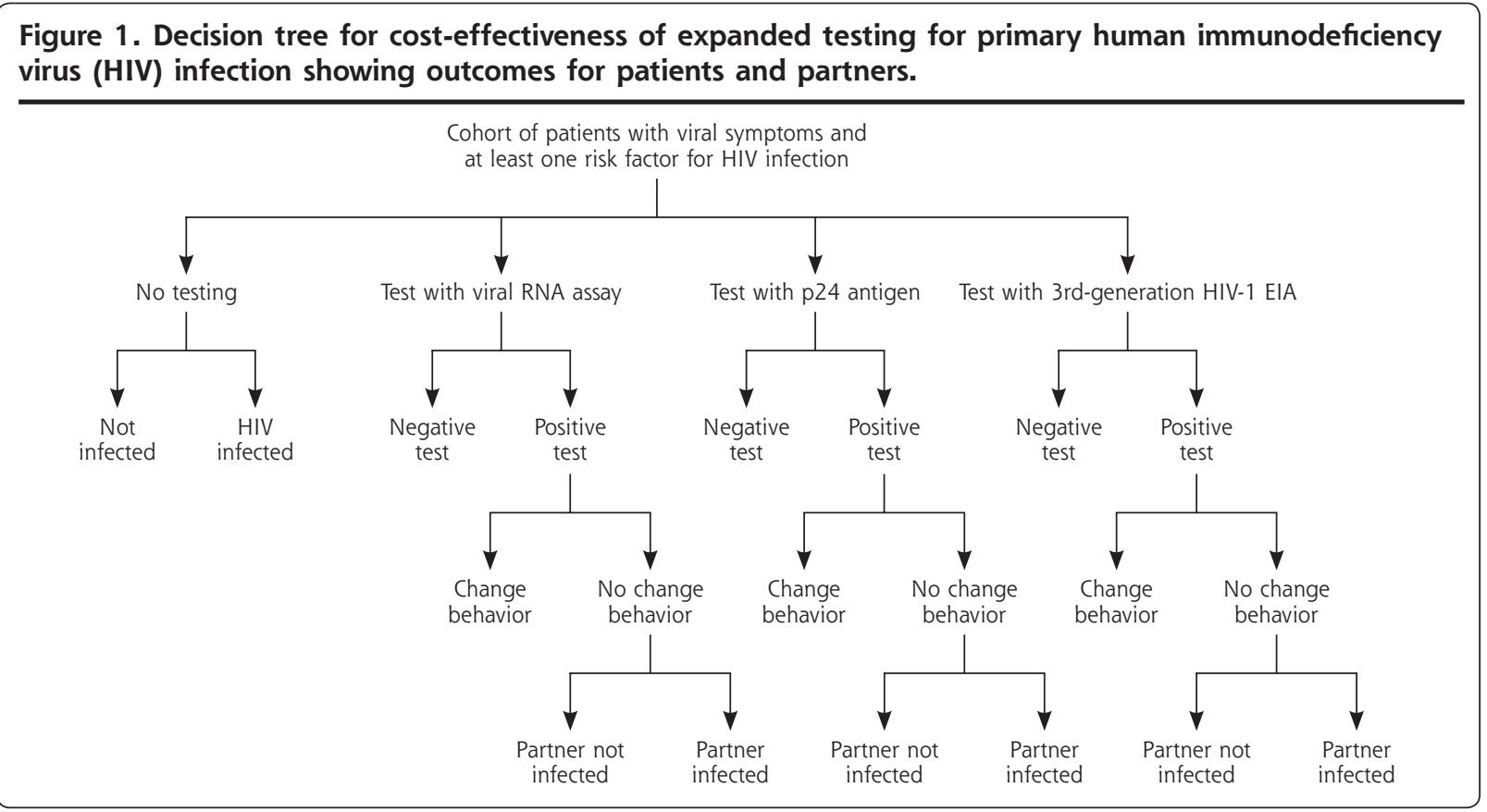




\begin{tabular}{|c|c|c|c|}
\hline Variable & $\begin{array}{l}\text { Baseline } \\
\text { Estimate }\end{array}$ & $\begin{array}{l}\text { Range Used in } \\
\text { Sensitivity Analysis }\end{array}$ & Source \\
\hline \multicolumn{4}{|l|}{ Costs (\$) } \\
\hline p24 antigen EIA & 24.65 & $12.33-49.30$ & Medicare fee schedule \\
\hline HIV-1 RNA assay & 118.89 & $59.45-237.78$ & Medicare fee schedule \\
\hline Third-generation HIV-1 EIA & 19.17 & $9.59-38.34$ & Medicare fee schedule \\
\hline Western blot assay & 27.05 & $13.53-54.10$ & Medicare fee schedule \\
\hline CD4 cell count per microliter & 90 & $45-180$ & Medicare fee schedule \\
\hline Initial battery of laboratorys tests (new diagnosis)* & 254 & $200-614$ & Medicare fee schedule \\
\hline Expanded testing program costs & 101.47 & $51-203$ & MMWR ${ }^{20}$ \\
\hline $\begin{array}{l}\text { Discounted lifetime medical costs (diagnosed with PHI and } \\
\text { antiretroviral therapy started at (D4 cell count of } 350 / \mu \mathrm{L} \text { ) }\end{array}$ & 95,800 & $47,900-191,600$ & Freedberg et a $\left.\right|^{23}$ \\
\hline $\begin{array}{l}\text { Discounted lifetime medical costs (PHI not diagnosed and } \\
\text { antiretroviral therapy started when HIV diagnosed) }\end{array}$ & 88,100 & $44,050-176,200$ & Freedberg et a $\left.\right|^{23}$ \\
\hline Return visit & 52.53 & $40-67.86$ & Kaplan \& Anderson ${ }^{24}$ \\
\hline \multicolumn{4}{|l|}{ Test characteristics } \\
\hline p24 antigen EIA, sensitivity & 0.887 & $0.770-0.957$ & Hecht et al ${ }_{1}^{8}$ Daar et al ${ }^{9}$ \\
\hline Specificity & 0.9996 & $0.9950-0.9999$ & Hecht et $a l_{1}^{8}$ Daar et al ${ }^{9}$ \\
\hline HIV-1 RNA, sensitivity & 1.000 & - & Hecht et al, ${ }^{8}$ Daar et al ${ }^{9}$ \\
\hline Specificity & 0.980 & $0.950-0.999$ & Hecht et al,,$^{8}$ Daar et al ${ }^{9}$ \\
\hline Third-generation HIV-1 EIA, sensitivity & 0.790 & $0.600-0.920$ & Hecht et $\left.a\right|^{8}$ \\
\hline Specificity & 0.970 & $0.930-0.990$ & Hecht et $\left.a\right|^{8}$ \\
\hline Probability of indeterminate Western blot & 0.000004 & - & Kleinman et $\mathrm{al}^{22}$ \\
\hline \multicolumn{4}{|l|}{ Prevalence factors (\%) } \\
\hline Patients lost to follow-up & 31 & $16-62$ & MMWR ${ }^{21}$ \\
\hline Prevalence in screened population & 0.66 & $0.53-0.92$ & Coco \& Kleinhans ${ }^{16}$ \\
\hline \multicolumn{4}{|l|}{ Sexual transmission factors } \\
\hline Patients who change behavior to avoid infecting sexual partner & 50 & $0-96$ & MMWR ${ }^{6}$ \\
\hline Patients that are sexually active & 50 & $25-85$ & MMWR ${ }^{6}$ \\
\hline Infectivity (probability of sexual transmission during PHI period) & 15 & $0-30$ & Yerly et $a_{1}{ }^{25}$ Pilcher et al ${ }^{26}$ \\
\hline \multicolumn{4}{|l|}{ Utilities } \\
\hline Asymptomatic HIV infection & 0.937 & $0.926-0.949$ & Schackman et al27 \\
\hline $\begin{array}{l}\text { Anxiety while waiting for confirmatory test results for patients } \\
\text { with a positive screen }\end{array}$ & 0.682 & $0.400-0.800$ & Kaplan \& Anderson ${ }^{24}$ \\
\hline \multicolumn{4}{|l|}{ Quality-adjusted life-expectancy (discounted), years } \\
\hline No PHI & 24 & - & $\mathrm{NCHS}^{15}$ \\
\hline Positive screening result, no $\mathrm{PHI}$ & 23.9735 & $23.950-23.983$ & $\begin{array}{l}\text { NCHS, }{ }^{15} \text { Kaplan \& } \\
\text { Anderson }{ }^{24}\end{array}$ \\
\hline $\begin{array}{l}\text { PHI diagnosed at screening with follow-up care and antiretroviral } \\
\text { treatment started at CD4 cell count of } 350 / \mu \mathrm{L}\end{array}$ & 11.9 & $11.832-11.952$ & Freedberg et $\mathrm{al}^{23}$ \\
\hline $\begin{array}{l}\text { PHI not diagnosed at screening or lost to care with antiretroviral } \\
\text { treatment started when HIV diagnosed }\end{array}$ & 11 & - & Freedberg et $\mathrm{a}^{23}$ \\
\hline
\end{tabular}

3-drug antiretroviral therapy was given according to Department of Health and Human Services guidelines, and patients incurred lifetime medical costs and survival times based on estimates from a mathematical simulation model of HIV disease. ${ }^{7,23}$ Additionally, some patients in this pathway altered their sexual behavior and thus avoided infecting their sexual partner during the several weeks of primary HIV infection symptoms. Partners who avoided infection avoided incurring lifetime HIV medical costs and reduced quality of life. Future behavior changes outside the symptomatic primary HIV infection period were not included in the analysis. Patients that failed to return for follow-up had HIV infection diagnosed at a later stage and incurred reduced lifetime medical costs and survival times based on an extrapolation of data from previous estimates of stage of disease when diagnosed, lifetime medical costs after starting combination antiretroviral therapy, and QALYs of survival. ${ }^{14,23}$

Patients in the false-positive category were assumed 
to have intermediate results on Western blot assay. Patients in this pathway were observed for 3 months with 2 follow-up visits. Testing confirmed a negative result after 2 standard HIV antibody tests, 3 Western blot assays, and a quantitative HIV-1 RNA assay.

Patients in the true-negative category had a followup visit with an HIV counselor to be informed of the negative results.

Patients in the false-negative category also had a brief follow-up visit with an HIV counselor to be informed of the negative results. These patients had HIV infection subsequently diagnosed at a later stage and incurred the same lifetime medical costs and survival times as those patients in the true-positive category who were lost to follow-up.

Patients that were not tested reported symptoms at later stages of infection, as did those patients in the true-positive category who were lost to follow-up.

The primary outcome measures were the incremental cost per QALY gained, the number of cases of primary HIV infection identified, and the threshold prevalence at which expanded testing had a cost per QALY of less than $\$ 50,000$, a value that is generally considered to be a cost-effectiveness threshold for a single patient. This analysis includes both patients and their partners; the ramifications of this approach are discussed below. Other outcomes were the number of cases of infection avoided through changes in sexual behavior, the number of false-positive diagnoses, the number of false-negative diagnoses, and the cost per case diagnosed. The model was programmed using decision analysis software (TreeAgePro [version 2004], TreeAge Software Inc, Williamstown, Mass).

\section{Decreased Transmission Through Change in Sexual Behavior}

From a public health perspective, determining which patients are in this phase of infection can decrease their high-risk behavior and have a substantial impact on subsequent transmission. In an unpublished study from the Centers for Disease Control and Prevention (CDC) of 1,363 HIV-infected men and women, among the $69 \%$ who were sexually active during the preceding 12 months, $78 \%$ to $96 \%$ used a condom at their most recent anal or vaginal intercourse with a known HIV-negative partner, and 52\%-86\% reported condom use with a partner of unknown serostatus. ${ }^{6}$ The analysis used lower rates of $50 \%$ for both estimates. To determine a rate of transmission, a study of 197 persons with documented primary HIV infection showed, through gene sequencing and contact tracing, that transmission occurred at the time of primary HIV infection in $30 \%{ }^{25}$ In keeping with a bias against expanded testing, the analysis decreased this rate by one half to $15 \%$, because HIV can be transmitted during the presymptomatic phase of primary HIV infection. ${ }^{26}$

\section{Data on Costs}

The costs of conducting an HIV-testing program were obtained from a state-funded program in Massachusetts that offered HIV counseling, testing, and referral to 3,068 patients entering 1 of 4 hospital-associated urgent care centers. ${ }^{20}$ Included in this program were the cost of HIV counselors and intake nurses who arranged for telephone follow-up, visits to homeless shelters, and travel vouchers to bring positive patients into care. The costs of laboratory tests were obtained from the Medicare fee schedule for Lancaster, Pa, for 2002. Clinical visit costs were obtained from a national survey of physician's office charges. ${ }^{30}$ Lifetime medical costs incurred after the initiation of 3-drug antiretroviral therapy at CD 4 cell counts of 350/ $\mathrm{LL}$ and lower were based an extrapolation of data from a computer-simulated model of HIV-infected persons. ${ }^{23}$ Costs were converted to 2002 dollars by the medical care component of the Consumer Price Index. ${ }^{31}$ Future costs were discounted at a rate of $3 \%$.

\section{Data on Health-Related Quality of Life}

Quality of life for asymptomatic HIV infection was obtained from a national probability sample of HIV infected adults. ${ }^{27}$ Utility values for the mental anguish resulting from waiting for confirmatory tests of a positive screening test were derived from the Quality of Well-Being index. ${ }^{24}$ QALYs for patients starting 3-drug antiretroviral therapy at CD 4 cell counts of $350 / \mu \mathrm{L}$ or less until time of death were based on an extrapolation of data from previous estimates of age and stage of disease presentation and QALYs of survival after initiation of combination antiretroviral therapy. ${ }^{14,23}$ Future gains in quality of life were discounted at a rate of $3 \%$.

\section{RESULTS \\ Base Case Analysis}

The base case analysis results are displayed in Table 2 . Expanded testing with the p24 antigen EIA test had the lowest incremental cost-effectiveness ratio of the 3 expanded testing strategies. Under base case assumptions, the cost per QALY for the p24 antigen EIA was $\$ 30,800$ compared with no testing. Testing with an HIV-1 RNA assay or the third-generation HIV-1 EIA was dominated, or evaluated as inferior, by the p24 EIA strategy.

The p24 antigen EIA strategy, because of the high specificity of the test, resulted in significantly fewer false-positive diagnoses: 1,127 compared with 90,257 and 59,169 for the third-generation EIA and HIV-1 
RNA assay, respectively (Table 2). The HIV-1 RNA assay, because of the high sensitivity of the test, identified 2,946 more cases of primary HIV infection and allowed for the avoidance of 66 more cases of infected partners compared with the p24 antigen EIA. Additionally, there were no false-negative cases with this option compared with 3,012 with the p24 antigen EIA option. The analysis also calculated costs from a third party payer perspective as testing cost per case identified. The p24 antigen EIA option had the lowest cost per case identified $(\$ 29,090)$.

\section{Sensitivity Analyses}

Threshold Analysis of Prevalence of Primary HIV Infection

To determine the lowest primary HIV infection prevalence at which expanded testing was cost-effective at the usual standard of $\$ 50,000$ per QALY, we performed a 1 -way sensitivity analysis (keeping all other variables constant) using the $95 \% \mathrm{CI}$ range of the directly measured prevalence estimate (0.1\%-1.9\%). ${ }^{17}$ Expanded testing with the p24 antigen EIA exceeded this standard when compared with no testing at a prevalence of less than $0.35 \%$. The incremental cost-effectiveness ratios for other prevalence rates reported in the literature, ${ }^{16,17}$ comparing expanded testing of primary HIV infection using the p24 antigen EIA with no testing, showed the following results: at a prevalence of $1 \%$ (urban patients with risk factors) costs were $\$ 23,000$ per QALY; at a prevalence of $0.56 \%$ (patients with a rash and other viral symptoms) costs were $\$ 35,000$ per QALY; at a prevalence of $0.13 \%$ (patients with a sore throat and other viral symptoms) costs were $\$ 129,000$ per QALY.

\section{Other 1-Way Sensitivity Analyses}

The baseline results remained fairly stable when compared with other 1 -way analyses using the variable ranges in Table 1. The p24 antigen EIA remained the most cost-effective option, with the cost per QALY, ranging from $\$ 15,500$ to $\$ 50,600$ when compared with no testing. The HIV-1 RNA assay continued to be inferior or have a cost per QALY of greater than $\$ 100,000$ compared with the p24 antigen EIA, and the thirdgeneration HIV-1 EIA remained inferior. The results of the key variables that had the largest impact on the baseline results are shown in Table 3 .

\section{Multiway Sensitivity Analyses}

We performed a Monte Carlo simulation (probabilistic sensitivity analysis) in which the values for all the variables listed in Table 1 were simultaneously varied. ${ }^{32}$ We entered each variable as a probability distribution based on reported $95 \% \mathrm{CIs}$, when available, or as a reasonable range as indicated in the third column in the table. The log-normal distribution was assumed for cost variables and the beta distribution for probability and utility variables. ${ }^{32}$ We randomly selected new values from within each of the probability distributions during each of 100,000 iterations and calculated 95\% likelihood comparisons of the strategies.

\section{Table 2. Cost, Effectiveness, and Incremental Cost-Effectiveness of Expanded Testing for Primary HIV Infection of 3,030,303 Hypothetical Patients at a Prevalence of $0.66 \%$ With Third-Generation HIV-1 ElA,} p24 Antigen EIA, and HIV-1 RNA Assay

\begin{tabular}{|c|c|c|c|c|}
\hline Variable & No Testing & $\begin{array}{c}\text { Third-Generation } \\
\text { HIV-1 EIA }\end{array}$ & $\begin{array}{l}\text { p24 Antigen } \\
\text { EIA }\end{array}$ & $\begin{array}{l}\text { HIV-1 } \\
\text { RNA Assay }\end{array}$ \\
\hline Cost (millions), \$ & $1,762.1$ & $2,233.6$ & $2,258.2$ & $2,561.8$ \\
\hline Incremental cost (millions), $\$$ & - & 471.5 & 24.6 & 303.6 \\
\hline Effectiveness (thousands) QALYs & $69,710.0$ & $69,720.8$ & $69,726.1$ & $69,725.8$ \\
\hline Incremental effectiveness, QALYs & - & 10,800 & 5,300 & $(300)$ \\
\hline \multicolumn{5}{|l|}{ Effectiveness, No. } \\
\hline Primary HIV infection cases diagnosed & - & 15,803 & 17,054 & 20,000 \\
\hline $\begin{array}{l}\text { Primary HIV infection cases lost to care } \\
\text { ( } 31 \% \text { of those diagnosed) }\end{array}$ & 20,000 & 4,899 & 5,287 & 6,200 \\
\hline False-positive diagnoses & - & 90,257 & 1,127 & 59,169 \\
\hline False-negative diagnoses & - & 2,924 & 3,012 & 0 \\
\hline Cases avoided per behavior change & & 403 & 435 & 501 \\
\hline \multicolumn{5}{|l|}{ Cost-effectiveness, $\$$} \\
\hline Testing cost per case identified & - & 29,836 & 29,090 & 39.985 \\
\hline $\begin{array}{l}\text { Incremental cost per quality-adjusted } \\
\text { year of life gained }\end{array}$ & - & Dominated* & 30,800 & Dominated* \\
\hline
\end{tabular}




\section{Table 3. Changes in Incremental Cost per Quality-Adjusted Life-Year in Key 1-Way Sensitivity Analyses}

1. Doubling the cost of lifetime medical care for patients being observed to CD4 cell counts of $350 / \mu \mathrm{L}$ or seeking care at later stages of infection $(\$ 88,100 / \$ 95,800$ to $\$ 176,050 / \$ 191,600)$ increased the cost of expanded testing with the p24 antigen EIA from $\$ 30,800$ to $\$ 34,100$ compared with no testing

2. Doubling the expanded testing and counseling enrollment program costs ( $\$ 101.47$ to $\$ 203$ ), increased the cost of the p24 antigen EIA testing option to $\$ 49,800$ compared with no testing

3. Increasing the specificity of the HIV-1 RNA assay (0.98 to 0.999) decreased the cost of the HIV-1 RNA assay option to $\$ 142,000$ compared with the p24 antigen EIA option

4. Assuming no benefit to sexual partners of patients with $\mathrm{PHI}$, ie, no cases avoided through changes in behavior, increased the cost of the p24 antigen EIA to $\$ 50,600$ when compared with no testing

$\mathrm{EIA}=$ enzyme immunosorbent assay; HIV = human immunodeficiency virus; $\mathrm{PHI}=$ primary HIV infection.

Figure 2. Probabilistic sensitivity analysis, p24 antigen EIA compared with no testing.

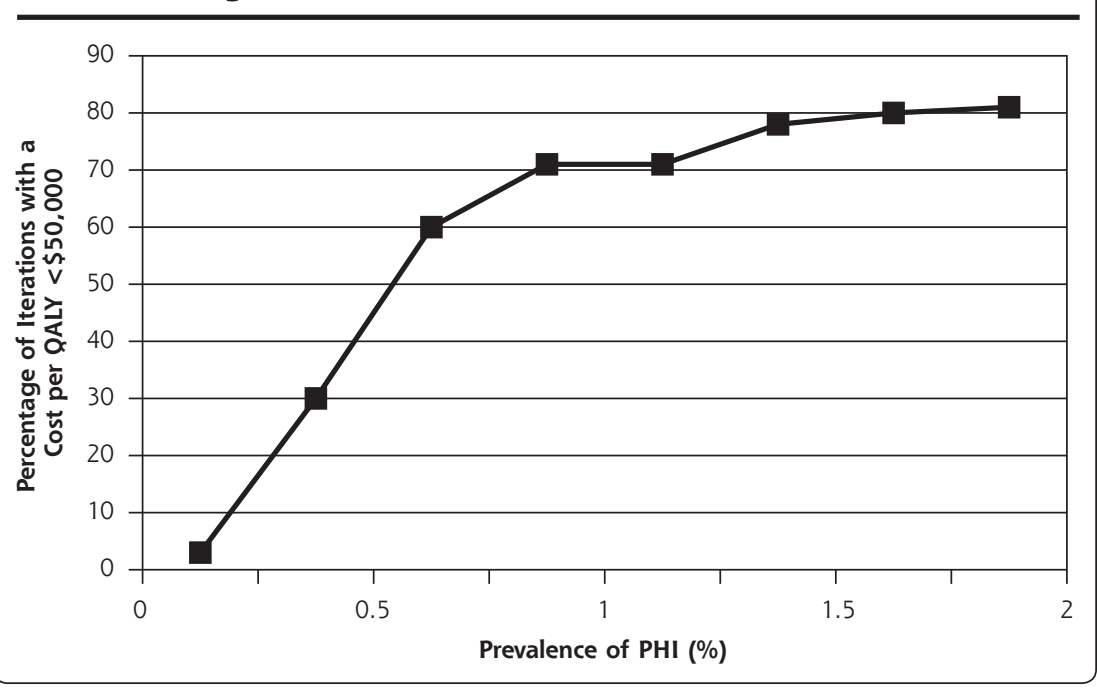

Figure 3. Probabilistic sensitivity analysis, HIV-1 RNA assay compared with p24 antigen EIA.

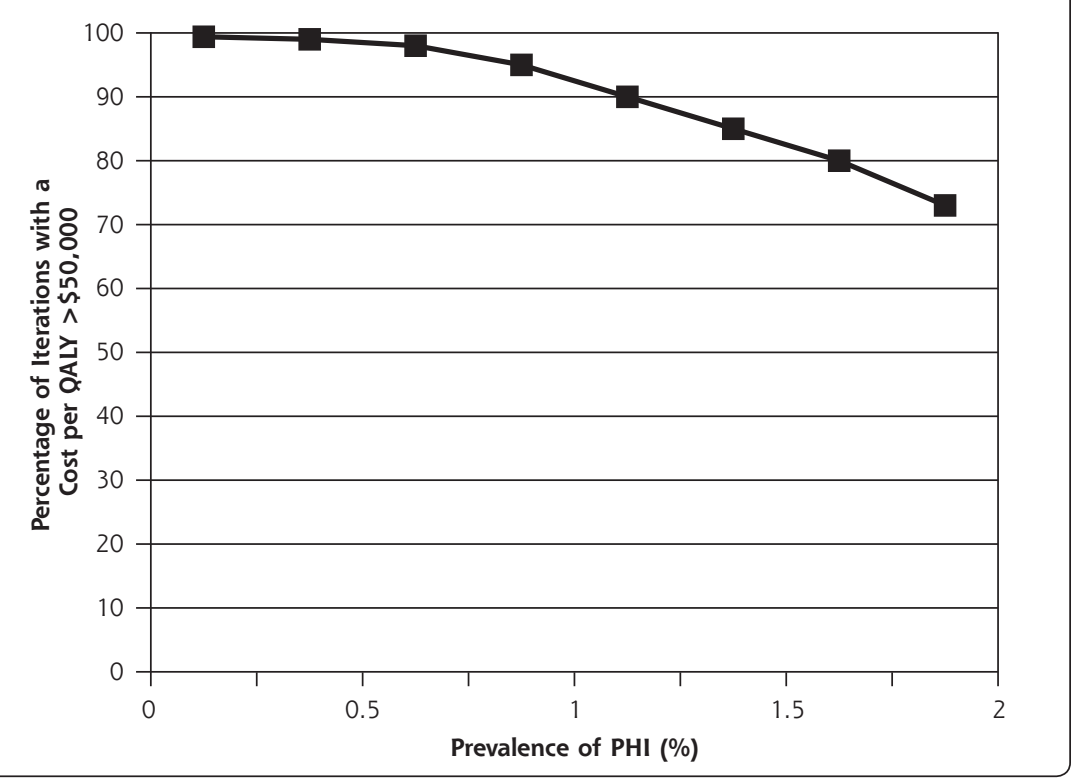

The p24 antigen EIA testing strategy had a $19 \%$ probability of being dominant (more effective and less costly), a $48 \%$ probability of having an a cost per QALY of less than $\$ 50,000$, and a $33 \%$ probability of having a cost per QALY of more than $\$ 50,000$ compared with no testing. There was a $0 \%$ chance of it being less effective. The HIV-1 RNA assay, when compared with the p24 antigen EIA, had a 3\% probability of being dominant or having a cost per QALY of less than $\$ 50,000$, a $44 \%$ probability of having a cost per QALY of more than $\$ 50,000$, and a $53 \%$ probability of being inferior (less effective and more costly). Figures 2 and 3 show how these comparison probabilities changed with varying the prevalence.

\section{DISCUSSION}

This study is the first to determine the cost-effectiveness of expanded testing for HIV infection in the acute phase. Using conservative assumptions, expanded testing of patients with fever, other viral symptoms, and at least 1 risk factor with the p2 4 antigen EIA had $67 \%$ probability of being superior or having a cost per QALY of less than $\$ 50,000$ compared with no testing. Because of the high specificity of the p24 antigen EIA, false-positive diagnoses would be limited to 1,127 of the $3,030,303$ persons tested in the model. Expanded testing would result in short-term avoidance of 435 infections in sexual partners. Although not modeled, early diagnosis of HIV infection could also allow for long-term decreased transmission, because patients would be aware of their communicable status for many more years. Also sexual partners 
who avoid infection would not transmit the infection to others. Consequently, expanded testing for primary HIV infection could have a strong impact on curtailing the HIV epidemic and contribute greatly to achieving the CDC goal of reducing the annual number of HIV infections by $50 \%$ per year by $2005 .{ }^{33}$

Several recent studies have addressed with varying results the issue of screening for HIV, not primary HIV infection, in the general population. ${ }^{34,35}$ One study found that 1 -time screening of the general population with a prevalence of $0.1 \%$ would cost $\$ 113,000$ per QALY, whereas another analysis found that screening populations with a prevalence as low as $0.05 \%$ had a cost per QALY of $\$ 50,000$ when including costs and benefits for partners. It is difficult to compare these results with this analysis of expanded testing for primary HIV infection because of the different screening costs and benefits to partners.

Our analysis has several limitations. The estimates of lifetime medical costs after initiation of antiretroviral therapy were derived from a study that used 1998 dollars for cost estimates. ${ }^{23}$ Although these costs were adjusted to 2002 dollars using the medical care component of the consumer price index, it is possible that the cost of antiretroviral therapy has increased faster than that of other medical costs. Again, however, doubling these costs through sensitivity analysis did not appreciably alter the incremental cost-effectiveness ratios of the screening options, because without any expanded testing program, HIV infection would be diagnosed at a later stage and would incur similar costs when treatment was started (Table 3).

The analysis was also limited by using test characteristics derived from previous studies that included patients who were recruited when they were outside the symptomatic phase of primary HIV infection. ${ }^{8,9}$ The sensitivity of the p24 antigen EIA, in particular, could have been underestimated in these studies. Our analysis was based on screening patients who sought care because of symptoms, which have an average duration of 14 days. $^{2}$ In a previous study, $100 \%$ of 20 patients with primary HIV infection tested within 1 week of symptom onset had positive p2 4 antigen EIA results, but they had negative results 3 weeks after symptom onset. ${ }^{36} \mathrm{~A}$ higher sensitivity estimate for the p24 antigen EIA would have resulted not only in a more favorable incremental cost-effectiveness ratio compared with no testing but also more cases detected and fewer false-negative diagnoses. Additionally, newer tests, such as fourth-generation HIV-1 EIAs and p24 antigen signal-amplification-boosted EIA of heat-denatured plasma, are now available that may perform better in screening for primary HIV infection, but have yet to be evaluated. ${ }^{37,38}$
The baseline prevalence estimate was derived from an analysis of a national outpatient database of patients with fever and other symptoms and diagnoses consistent with primary HIV infection. ${ }^{16}$ That estimate was based on assumptions that may not reflect the actual number of patients with primary HIV infection seeking medical care. Until more accurate data are available on the prevalence of primary HIV infection in low-risk populations, caution should be exercised in developing expanded testing policy based on these results. This analysis, however, showed that expanded testing for primary HIV infection has a $60 \%$ probability of being cost-effective at a prevalence of $0.35 \%$, or almost one half of the baseline rate.

Finally, cost-effectiveness analyses typically apply the $\$ 50,000$ per QALY standard to individual patients. This analysis combined the QALYs of the patients being tested and their sexual partners who avoided infection because of the communicable nature of HIV infection to show the full impact of an expanded testing program. In 1-way sensitivity analysis it was shown that without preventing transmission to sexual partners, the p24 antigen EIA had a cost per QALY of $\$ 50,600$ compared with no testing (Table 3 ).

Using reasonable assumptions, the analysis has shown that expanded testing for primary HIV infection with the p24 antigen EIA has a high probability of being cost-effective. The cost-effectiveness of expanded testing for primary HIV infection in populations with a prevalence of $0.66 \%$ or greater compares favorably with accepted screening programs, such as colon cancer screening, annual Papanicolaou smears in HIV-infected women, and breast cancer screening. ${ }^{39-41}$ Expanded testing for primary HIV infection may be a sound expenditure of health care resources and could have an impact on curtailing the HIV epidemic in the United States.

To read or post commentaries in response to this article, see it online at http://www.annfammed.org/cgi/content/full/3/5/391.

Key words: HIV infections/prevention $\&$ control; cost-benefit analysis; mass screening; delivery of health care; health services research

Submitted March 23, 2004; submitted, revised, December 8, 2004; accepted December 22, 2004.

\section{References}

1. Kahn JO, Walker BD. Acute human immunodeficiency virus type 1 infection. N Engl J Med. 1998;339:33-39.

2. Schacker T, Collier AC, Hughes J, Shea T, Corey L. Clinical and epidemiologic features of primary HIV infection. Ann Intern Med. 1996; 125:257-264.

3. Flanigan T, Tashima KT. Diagnosis of acute HIV infection: it's time to get moving! Ann Intern Med. 2001;134:75-77.

4. Gates W, Chesney MA, Cohen MS. Primary HIV infection -a public health opportunity. Am J Pub Health. 1997;87:1928-1930. 
5. Jacquez JA, Koopman JS, Simon CP, Longini IMJ. Role of the primary infection in epidemics of HIV infection in gay cohorts. J Acquir Immune Defic Syndr. 1994;7:1169-1184.

6. Centers for Disease Control and Prevention (CDC). Adoption of protective behaviors among persons with recent HIV infection and diagnosis-Alabama, New Jersey, and Tennessee, 1997-1998. MMW/R Morb Mortal W/kly Rep. 2000;49:512-515.

7. Guidelines for the Use of Antiretroviral Agents in HIV-1-Infected Adults and Adolescents. Washington, DC: Panel on Clinical Practices for Treatment of HIV Infection convened by the Department of Health and Human Services (DHHS). Available at: http://www.aidsinfo.nih.gov/guidelines.

8. Hecht FM, Busch MP, Rawal B, et al. Use of laboratory tests and clinical symptoms for identification of primary HIV infection. AIDS. 2002; 16:1119-1129.

9. Daar ES, Little S, Pitt J, et al. Diagnosis of primary HIV-1 infection. Los Angeles County Primary HIV Infection Recruitment Network. Ann Intern Med. 2001;134:25-29.

10. Royce RA, Sena A, Cates W, Jr., Cohen MS. Sexual transmission of HIV. N Engl J Med. 1997;336:1072-1078.

11. MacKellar DA, Valleroy LA, Secura GM, et al. Unrecognized HIV infection, risk behaviors, and perceptions of risk among young men who have sex with men: opportunities for advancing HIV prevention in the third decade of HIVIAIDS. J Acquir Immune Defic Syndr. 2005;38:603-614.

12. Dybul M, Fauci AS, Bartlett JG, Kaplan JE, Pau AK. Guidelines for using antiretroviral agents among HIV-infected adults and adolescents. Ann Intern Med. 2002;137:381-433.

13. Fagard C, Oxenius A, Gunthard $H$, et al. A prospective trial of structured treatment interruptions in human immunodeficiency virus infection. Arch Intern Med. 2003;163:1220-1226.

14. Klein D, Hurley LB, Merrill D, Quesenberry CP, Jr. Review of medical encounters in the 5 years before a diagnosis of HIV- 1 infection: implications for early detection. J Acquir Immune Defic Syndr. 2003;32:143-152.

15. National Center for Health Statistics. Vital Statistics. Available at: http://www.cdc.gov/nchs/data/nvsv.

16. Coco AS, Kleinhans E. The prevalence of primary HIV infection in symptomatic ambulatory patients. Ann Fam Med. 2005;3:400-404.

17. Pincus JM, Crosby SS, Losina E, et al. Acute human immunodeficiency virus infection in patients presenting to an urban urgent care center. Clin Infect Dis. 2003;37:1699-1704.

18. Vu MQ, Steketee RW, Valleroy L, et al. HIV incidence in the United States, 1978-1999. J Acquir Immune Defic Syndr. 2002;31:188-201.

19. Karon JM, Fleming PL, Steketee RW, De Cock KM. HIV in the United States at the turn of the century: an epidemic in transition. Am J Public Health. 2001;91:1060-1068.

20. Centers for Disease Control and Prevention (CDC). Voluntary HIV testing as part of routine medical care--Massachusetts, 2002. MMWR Morb Mortal W/kly Rep. 2004;53:523-526.

21. Advancing HIV prevention: new strategies for a changing epidemicUnited States, 2003. MMWR Morb Mortal W/kly Rep. 2003;52:329-332.

22. Kleinman S, Busch MP, Hall L, et al. False-positive HIV-1 test results in a low-risk screening setting of voluntary blood donation. Retrovirus Epidemiology Donor Study. JAMA. 1998;280:1080-1085.
23. Freedberg KA, Losina $E$, Weinstein $M C$, et al. The cost effectiveness of combination antiretroviral therapy for HIV disease. $N$ Engl I Med. 2001;344:824-831.

24. Kaplan RM, Anderson JP. A general health policy model: update and applications. Health Serv Res. 1988;23:203-235.

25. Yerly S, Vora S, Rizzardi P, et al. Acute HIV infection: impact on the spread of HIV and transmission of drug resistance. AIDS. 2001;15:2287-2292.

26. Pilcher $C D$, Eron JJ, Jr., Vemazza $\mathrm{PL}$, et al. Sexual transmission during the incubation period of primary HIV infection. JAMA. 2001;286:1713-1714.

27. Schackman BR, Goldie SJ, Freedberg KA, et al. Comparison of health state utilities using community and patient preference weights derived from a survey of patients with HIVIAIDS. Med Decis Making. 2002;22:27-38.

28. Kaplan JE, Masur H, Holmes KK. Guidelines for preventing opportunistic infections among HIV-infected persons--2002. Recommendations of the U.S. Public Health Service and the Infectious Diseases Society of America. MMWR Recomm Rep. 2002;51:1-52.

29. Fauci AS, Pantaleo G, Stanley S, Weissman D. Immunopathogenic mechanisms of HIV infection. Ann Intern Med. 1996;124:654-663.

30. Wasserman Y. Physicians' Fee Reference 2002. West Allis, Wisc: DMD Medical Publishers, Ltd; 2002

31. Medical Consumer Price Index. Available at: http://www.cdc.gov/ nchs/data/hus/tables/2003.

32. Briggs AH. Handling uncertainty in cost-effectiveness models. Pharmacoeconomics. 2000;17:479-500

33. Holtgrave DR, Pinkerton SD. Economic implications of failure to reduce incident HIV infections by $50 \%$ by 2005 in the United States. J Acquir Immune Defic Syndr. 2003;33:171-174.

34. Paltiel AD, Weinstein MC, Kimmel AD, et al. Expanded screening for HIV in the United States--an analysis of cost-effectiveness. $N$ Engl J Med. 2005;352:586-595.

35. Sanders GD, Bayoumi AM, Sundaram V, et al. Cost-effectiveness of screening for HIV in the era of highly active antiretroviral therapy. $N$ Engl J Med. 2005;352:570-585.

36. Lindback S, Karlsson AC, Mittler J, et al. Viral dynamics in primary HIV-1 infection. Karolinska Institutet Primary HIV Infection Study Group. AIDS. 2000; 14:2283-2291.

37. Weber B, Fall EH, Berger A, Doerr HW. Reduction of diagnostic window by new fourth-generation human immunodeficiency virus screening assays. J Clin Microbiol. 1998;36:2235-2239.

38. Schupbach J. Measurement of HIV-1 p24 antigen by signal-amplification-boosted ELISA of heat-denatured plasma is a simple and inexpensive alternative to tests for viral RNA. AIDS Rev. 2002;4:83-92.

39. Pignone M, Saha S, Hoerger T, Mandelblatt J. Cost-effectiveness analyses of colorectal cancer screening: a systematic review for the U.S. Preventive Services Task Force. Ann Intern Med. 2002;137:96-104

40. Goldie SJ, Weinstein MC, Kuntz KM, Freedberg KA. The costs, clinical benefits, and cost-effectiveness of screening for cervical cancer in HIV-infected women. Ann Intern Med. 1999;130:97-107.

41. Salzmann P, Kerlikowske K, Phillips K. Cost-effectiveness of extending screening mammography guidelines to include women 40 to 49 years of age. Ann Intern Med. 1997;127:955-965. 